\title{
Near-field self-induced hollow spot through localized heating of polycarbonate/ZnS stack layer
}

\author{
Alberto da Costa Assafrao, ${ }^{\star}$ Arthur J. H. Wachters, \\ Silvania F. Pereira, and H. Paul Urbach \\ Optics Research Group, Department of Imaging Science and Technology, Delft University of Technology, \\ Lorentzweg 1, CJ Delft 2628, The Netherlands \\ *Corresponding author: a.dacostaassafrao@ tudelft.nl \\ Received 13 September 2012; accepted 10 October 2012; \\ posted 15 October 2012 (Doc. ID 176160); published 30 October 2012
}

\begin{abstract}
We have found an alternative way of achieving a doughnutlike focused spot by simply melting a subwavelength scatterer in a polycarbonate/ZnS sample. The near-field microscopy technique is used to directly measure the induced doughnut spot in the near-field regime. A numerical model based on rigorous solution of the Maxwell's equations is proposed to study the phenomena. The simulations help to understand the optical mechanism behind the spot formation. (C) 2012 Optical Society of America

OCIS codes: $\quad 190.0190,190.4870$.
\end{abstract}

\section{Introduction}

In many modern optical techniques, there is an increasing interest in having a structured focused spot in the focal plane of an optical system. In particular, a doughnutlike focused spot is desired because of its interesting properties and potential applications. For example, stimulated emission depletion microscopy relies on a hollow spot, i.e., the dark central region of a doughnut-shaped spot, to achieve superresolution [1]. Double-beam subdiffraction lithography using two beams and direct laser writing also use a doughnutlike spot [2,3]. Another fascinating application is optical trapping, where the central dark zone of the doughnut spot is used to trap and manipulate tiny objects that are repelled and pushed away from the regions of maximum intensity [4,,$\underline{5}]$.

A doughnutlike spot can be generated by manipulating the state of polarization of a laser beam incident on a lens. Particularly, an azimuthally polarized light leads to such hollow spots [6,7]. When focusing an azimuthally polarized beam, a dark central zone is always achieved due to a nonexistence of a

$1559-128 \mathrm{X} / 12 / 317684-06 \$ 15.00 / 0$

(C) 2012 Optical Society of America longitudinal field component at the focal plane, regardless of the NA of the focusing lens [8]. Typically, additional optical elements are required along the optical lightpath in order to change the state of polarization of the light $[6,9]$, which inevitably raises the complexity of the optical setup, and in most cases, the final cost of the setup. In addition, by placing a polarizer along the optical path, a considerable amount of light energy is lost, which may be disadvantageous in some applications. In this context, it becomes interesting to have an alternative technique that generates a doughnutlike focused spot without changing the polarization of a laser beam.

In this work, we aim to experimentally demonstrate the possibility of having a near-field structured doughnut focused spot by simply using a sample composed of polycarbonate (PC) substrate and a thin layer of a $\mathrm{ZnS}: \mathrm{SiO}_{2}$. The basic idea behind this technique, which we call near-field self-induced hollow spot, is to focus a laser through the PC layer onto the $\mathrm{ZnS}: \mathrm{SiO}_{2}$, in such a way that part of the light reflected from the interface $\mathrm{PC} / \mathrm{ZnS}: \mathrm{SiO}_{2}$ constructively interferes in the vicinity of the boundary, already in the PC layer, heating a small portion of it. As a result, this tiny region will degrade into a molten state, resulting in a change in the optical 
parameters, as if a near-field optical scatterer were created in the PC layer. The incoming focused light generating and interacting with this near-field scatterer would result in a localized near-field hollow spot. To investigate whether a doughnutlike spot is indeed generated, near-field measurements of the focused light transmitted through the sample are performed using a scanning near-field optical microscope (SNOM), with a near-field probe in direct contact with the surface of the sample. In addition, a phenomenological model supported by experimental evidence is developed and run on a rigorous threedimensional (3D) finite-element method (FEM) tool $[10,11]$ to describe the effect. Finally, the model is employed to investigate another type of material, namely, silver dioxide, which potentially yields to a similar structured spot formation. We divide this paper in two main sections. In the first one, Section 2 , the details of the experimental setup, along with the near-field measurements, are shown. In the second part, Section 3 , we present the numerical model, based on rigorous solution of the Maxwell's equations, useful to understand the measurement results. Conclusions of this work are given in Section $\underline{4}$.

\section{Experimental Generation of the Doughnut Spot}

\section{A. Experimental Setup}

A schematic diagram of the experimental setup is shown in Fig. 1. A diode laser operating at $405 \mathrm{~nm}$ wavelength (Laser Components, CS4051205X) was coupled into a single-mode fiber and redirected to the SNOM (Witec Alpha 300S). Modifications were done in the SNOM scanning table to include a NA = 0.6 DVD lens, mounted on a $20 \mu \mathrm{m}$ piezo stage (Jena Mipos 20), so that the focal plane can be adjusted.

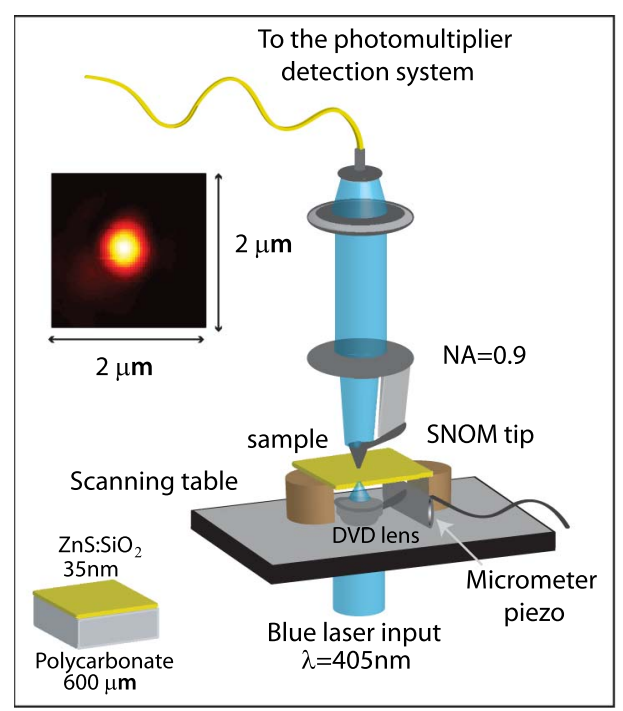

Fig. 1. (Color online) Lightpath inside the SNOM microscope. The modified scanning table holds the DVD lens and the sample. Near-field measurements are taken with the SNOM tip in contact with the sample surface, in the near-field regime. An example of a measured focused spot is shown on the top left.
The sample was placed in the focal region of the DVD lens. Near-field maps of the focused spots were taken in a $2 \mu \mathrm{m} \times 2 \mu \mathrm{m}$ scanning window. In this study, we have used a sample consisting of one layer of $\mathrm{ZnS}: \mathrm{SiO}_{2}$ (35 nm thick) deposited on a $600 \mu \mathrm{m}$ PC substrate, as shown in the bottom left of Fig. 1. A second sample consisting of $35 \mathrm{~nm} \mathrm{ZnS}: \mathrm{SiO}_{2}$ deposited on glass by magnetron sputtering was also fabricated for reference.

\section{B. Near-Field Measurements}

The spot measurements were carried out in the nearfield regime, i.e., with the SNOM probe in contact with the sample surface. The laser light is focused on the sample. In order to determine the best focal plane, an initial set of measurements at low power was performed. The smallest spot with highest intensity is considered as the best focused spot. An example of a measured spot in the best focal plane is shown on the top left of Fig. 1. The spot full width at half maximum is found to be $385 \pm 5 \mathrm{~nm}$, slightly higher than the theoretical diffraction-limited spot of the system, $340 \mathrm{~nm}$.

After finding the focal plane, the laser intensity was gradually increased, in steps of $0.5 \mathrm{~mW}$, and for each step a new near-field measurement was taken, always in the same plane. In Fig. 2, a sequence of measured spots is shown for laser intensities varying from 2.0 to $3.5 \mathrm{~mW}$. At $2.0 \mathrm{~mW}$, the focused spot presents the expected Airy pattern. After increasing the intensity, a disruption on the spot Airy-like intensity profile is gradually observed. The spots measured at 2.5 and $3.0 \mathrm{~mW}$ exemplify this gradual progression, which, at a final stage, turns into a doughnutlike focused spot, as seen in the bottom right of the figure.

Once the doughnut spot is observed, its characteristics are studied. The diameter of the doughnut spot is found to be approximately $1.1 \mu \mathrm{m}$, whereas the diameter of the inner dark region is around $780 \mathrm{~nm}$,
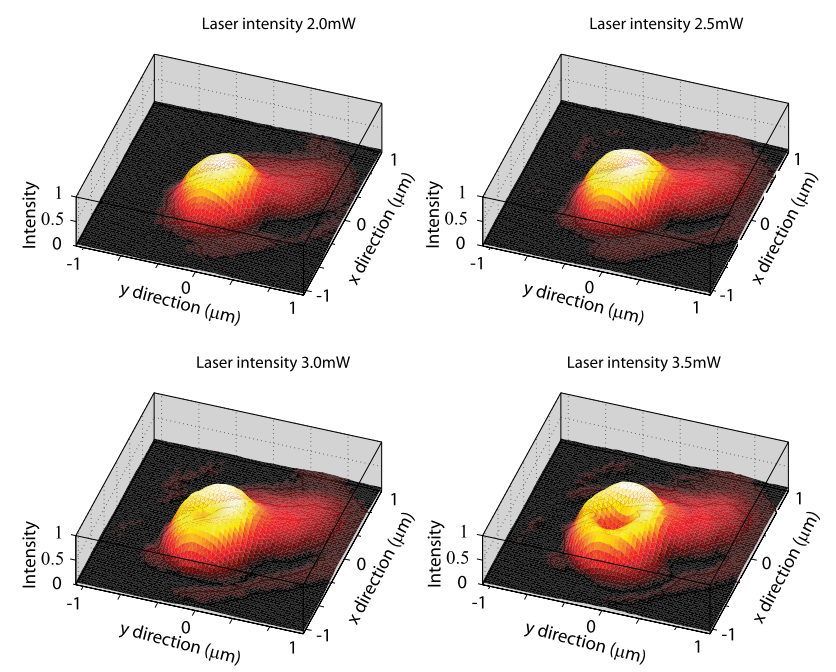

Fig. 2. (Color online) Measured focused spots intensity distribution at the focal plane for different laser power. The doughnut spot is formed at $3.5 \mathrm{~mW}$. 

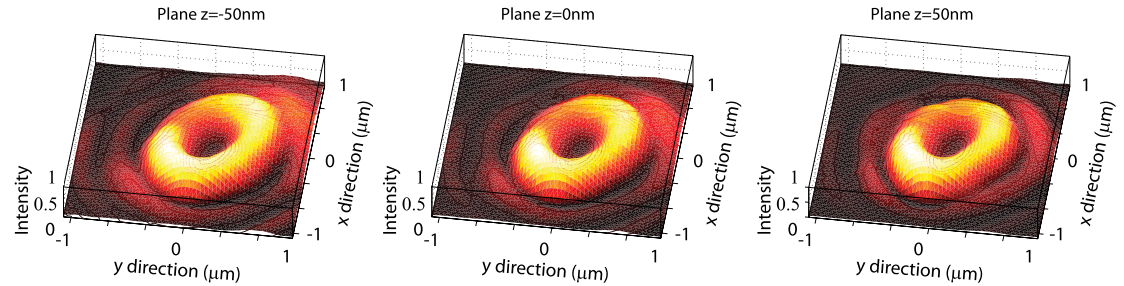

Fig. 3. (Color online) Measured intensity distribution of the doughnut spots at different planes. After its formation, the doughnut-shaped spot does not change significantly after defocusing the DVD lens.

at the best focal plane. By slightly adjusting the DVD lens to focus either before or after the best focal plane, no significant changes in the spot shape were observed, as displayed in Fig. 3 . These results indicate a reasonably good stability of the formed spot.

To exclude the possibility of having the $\mathrm{ZnS}: \mathrm{SiO}_{2}$ layer as the source of the effect, we performed the same type of measurements on the reference sample, in which the $\mathrm{ZnS}: \mathrm{SiO}_{2}$ layer is deposited on a glass substrate. None of the measurements resulted in the referred doughnut spot, regardless of the laser power employed. In Fig. 4, we show the peak intensity values as function of the laser power, obtained from the measured focused spots using the PC and reference samples. Note that the maximum intensity on the reference sample always occurs at the center of the Airy-like spot profile, whereas in the PC sample it may be shifted to the rings of the doughnut after its formation. Clearly, the peak intensity of the measured spots on the glass sample increases linearly with the laser power. This linear behavior does not occur in the PC sample, which indicates that the

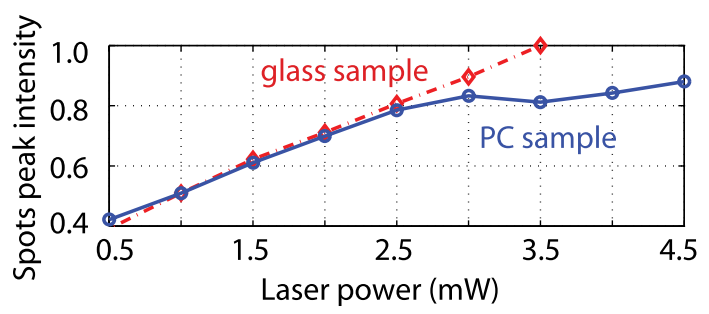

Fig. 4. (Color online) Focused spots peak intensity value as function of the laser power. A linear response was obtained with the reference sample, indicating that the nonlinear response is coming from the PC. optical properties of the PC layer must have changed. In particular, the decrease in the peak value is an indication that the extinction coefficient of the PC refractive index has increased during the process. In the next section we will explore this possibility using a simulation model in an attempt to understand the near-field doughnut spot formation.

\section{Simulation Model}

\section{A. Field on the Focal Plane}

Prior to a fully rigorous 3D FEM model, we have computed the focused spot in a stratified focal region by solving the vectorial diffraction integral using the Richards and Wolf method (see [12] and the references therein), for the PC with and without the $\mathrm{ZnS}: \mathrm{SiO}_{2}$ layer. The $\mathrm{NA}=0.6$ objective lens focuses a circularly polarized laser beam, from the positive $z$ direction, at the plane $z=0 \mathrm{~nm}$ (i.e., inside the $\mathrm{ZnS}: \mathrm{SiO}_{2}$ layer). The refractive indices of the $\mathrm{PC}$ and the $\mathrm{ZnS}: \mathrm{SiO}_{2}$ are $n=1.62$ and $n=2.21$ at $405 \mathrm{~nm}$, respectively [13]. The results are shown in Fig. 5. In both cases, the light reflected from the boundaries $\mathrm{PC} / \mathrm{ZnS}: \mathrm{SiO}_{2}$ and PC/air interferes with the incoming light, generating the standing waves seen in the positive $z$ region of the space. However, when the $\mathrm{ZnS}: \mathrm{SiO}_{2}$ is present, the computed peak intensity of the first maxima is enhanced by a factor of 1.5 when compared with the case where the $\mathrm{ZnS}: \mathrm{SiO}_{2}$ is absent. Based on this observation, it is plausible to conclude that, in a tiny overheated region close to the boundary $\mathrm{PC} / \mathrm{ZnS}: \mathrm{SiO}_{2}$ where this peak intensity occurs, a thermal induced amorphization of the $\mathrm{PC}$ material would result in changes in the electric permittivity.

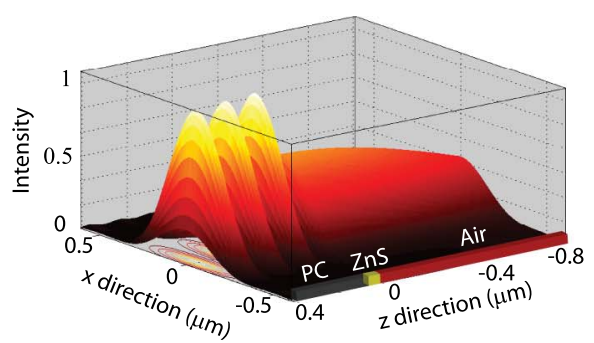

(a)

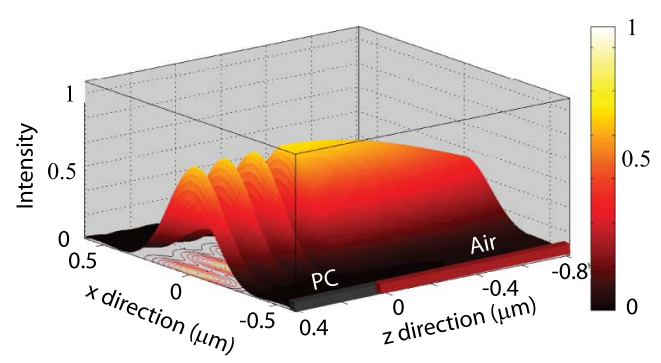

(b)

Fig. 5. (Color online) Computed focused electric field distribution in a multilayered space consisting of (a) PC/ZnS/air and (b) PC/air. Light reflected from the interfaces interferes constructively with the incoming light. Higher peak will occur when the $\mathrm{ZnS}_{\mathrm{SiO}} \mathrm{Sis}_{\mathrm{present}}$. The intensities are normalized by the maximum value of (a). 


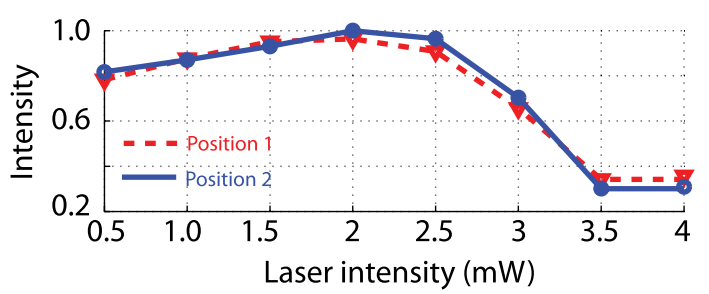

Fig. 6. (Color online) Measured intensity values at the coordinates coincident with the center of the formed hollow spot. The intensity drops from the unity to approximately 0.3 , for nonmolten to molten PC. Positions 1 and 2 refer to two neighbor pixels in the measured data.

\section{B. Rigorous 3D Model}

Following the discussion from the previous subsection, there is evidence that a change in the refractive index of the PC layer will occur in a tiny region close to the interface with the $\mathrm{ZnS}: \mathrm{SiO}_{2}$ layer. Therefore, to model such an effect, we assume that the permittivity function of the PC layer becomes a function of position, $\epsilon=\epsilon(x, y, z)$, to represent the local thermally modified region. The generation of this modified region occurs at places where the standing waves have high intensity values. For simplicity, we consider that only the first maxima in the PC layer (the one closest to the $\mathrm{ZnS}: \mathrm{SiO}_{2}$ layer) will generate the modified region. The physical dimensions of the modified region can be estimated as follows: along the $z$-axis, the first maxima is localized at $z=95 \mathrm{~nm}$ and the normalized intensities within the range $75 \leq z \leq$ $115 \mathrm{~nm}$ are higher than 0.8 . On the radial direction, the intensity profile has approximately a Gaussian distribution, which in turn has circular symmetry in the $x-y$ plane. Therefore, we define the following permittivity function for the PC layer:

$$
\begin{aligned}
\epsilon & =\epsilon(x, y, z=115 \leq z \leq 75) \\
& = \begin{cases}\left(n_{2}+k_{2} i\right)^{2}, & \text { if } \sqrt{x^{2}+y^{2}} \leq r, \\
\left(n_{1}+k_{1} i\right)^{2}, & \text { elsewhere }\end{cases}
\end{aligned}
$$

where $r$ is the radius of the circular region within the focused spot whose intensity values are above a chosen threshold, exemplarily considered as 0.8 . Increasing the laser intensity is emulated by

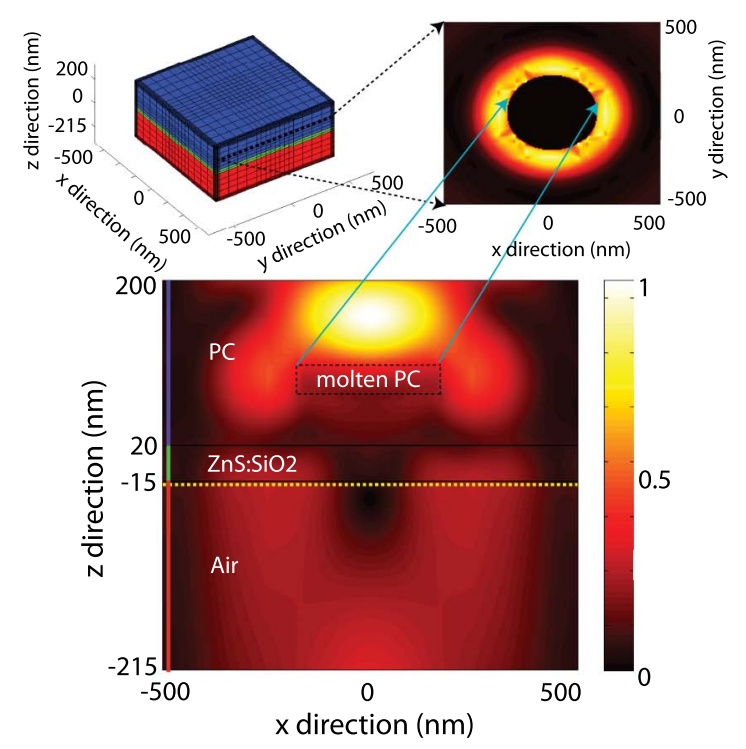

(a)

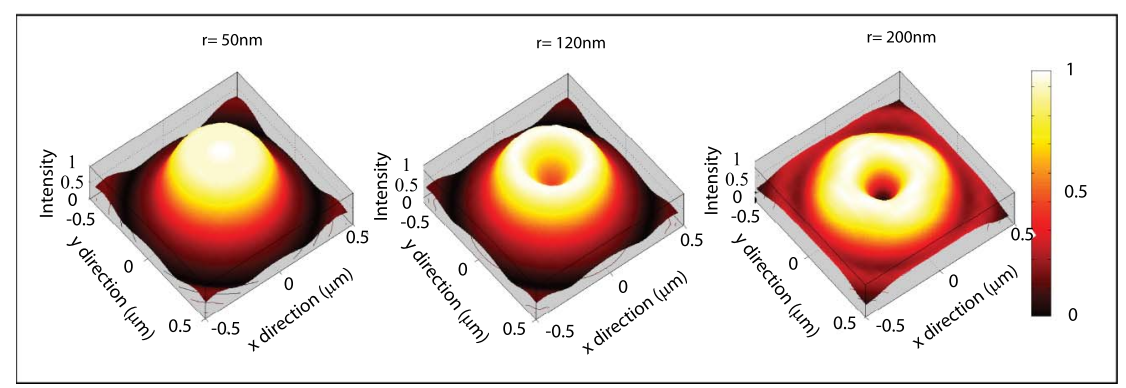

(b)

Fig. 7. (Color online) Schematics of the proposed model. (a) Top left: 3D FEM computational box with refinement in the region where the cylinder has to be meshed. Top right: the actual representation of the cylinder projected in the $x-y$ plane. The cylinder is colored dark on purpose to enhance visibility. Bottom figure: normalized total electric field along the $x-z$ plane for the complete stack layer, including the molten region with $r=120 \mathrm{~nm}$. A dark spot is formed underneath the $\mathrm{ZnS}_{-} \mathrm{SiO}_{2}$ layer. (b) Simulated focused spot across the $x-y$ plane, $5 \mathrm{~nm}$ below the $\mathrm{ZnS}-\mathrm{SiO}_{2}$ /air boundary. A clear doughnut spot formation is verified. 
changing the threshold value, and consequently, the radius $r$ and the $z$ interval. A quick analysis of Eq. (1) reveals that, in practice, a cylinder with radius $r$ and height equal to the $z$-axis interval is defined in a small region inside the focused spot area. The permittivity is taken as the squared refractive index, whose values in the molten state should be such that the inner part of the cylinder becomes more absorbing than the outside part, resulting in a structure that can be referred to as "near-field scatterer structure."

Next, we estimate the PC refractive index in the molten state. For this goal, we have considered the (complete) set of measurements shown in Fig. 2 . The intensity values at the coordinates coincident with the center of the formed doughnut spot are measured and plotted in Fig. 6 . The measured intensity drops from the unity to approximately 0.3 , in the center of the hollow spot (position 1), which is formed underneath the molten region. Next, we assumed a molten PC layer with thickness of $40 \mathrm{~nm}(115 \leq z \leq$ $75 \mathrm{~nm}$ ), and computed the transmitted focused field intensity using the Richards and Wolf approach. The molten PC layer is assumed to have the same real part of the refractive index as in the initial state, whereas the imaginary part was varied in order to match the measured drop in intensity. Under these conditions, we roughly estimate that the extinction coefficient that allows for such an intensity drop should be approximately $k_{2}=2$.
In Fig. 7(a), a schematic drawing of the proposed rigorous model is shown. A 3D FEM computational box is defined on a hexahedral mesh, refined in the region where the cylinder has to be meshed, as seen in the top left. On the top right, the actual representation of the circular projection of the cylinder is emphasized. In the bottom figure, the normalized total electric field, computed along the $x-z$ plane for the complete stack layer, including the molten region $(r=120 \mathrm{~nm})$, is shown. The $z$-axis shows the position of each layer as they are defined in the computational domain. As seen, the molten region in the PC layer (dotted square) is responsible for absorbing part of the incident light, leaving a dark region underneath it. Below the $\mathrm{ZnS}-\mathrm{SiO}_{2}$ layer, the dark region can still be seen.

To evaluate the spot field distribution across the $x-y$ plane, we computed the electric field in the plane $5 \mathrm{~nm}$ below the $\mathrm{ZnS}_{-} \mathrm{SiO}_{2} /$ air boundary (in the $z$ direction) for different radius $r$, as shown in Fig. 7(b). This particular $z$-plane is chosen to allow for qualitative comparisons with the near-field profiles measured experimentally with the SNOM. When the scatterer region has a small radius, $r=50 \mathrm{~nm}$, a small portion in the central region of the incident spot is partially absorbed. However, no doughnut formation is seen. As the radius of the scatterer region is increased, a clear doughnut-shaped intensity distribution is formed. A larger scatterer, having a radius of $200 \mathrm{~nm}$, results in a hollow spot with a very

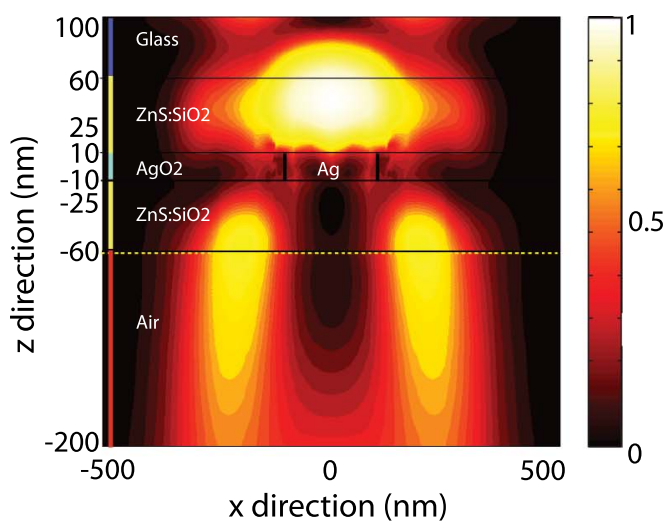

(a)

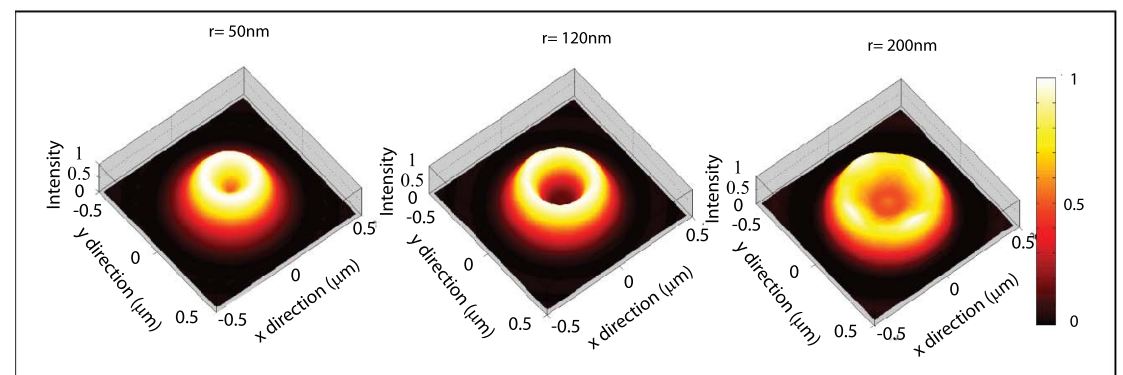

(b)

Fig. 8. (Color online) (a) Normalized total electric field along the $x-z$ plane for the complete $\mathrm{AgO}_{x}$ stack layer, including the Ag cluster region with $r=120 \mathrm{~nm}$. A dark spot is again formed underneath the $\mathrm{ZnS}-\mathrm{SiO}_{2}$ layer. (b) Simulated focused spot across the $x-y$ plane, $5 \mathrm{~nm}$ below the $\mathrm{ZnS}-\mathrm{SiO}_{2}$ /air boundary. The desired doughnut spot formation is observed. 
dark central region. The diameter of the doughnut spot is $920 \mathrm{~nm}$, and the diameter of the inner dark region is $500 \mathrm{~nm}$. After this point, the doughnut shape deteriorates gradually, being completely lost if the radius of the scatterer is comparable or larger than the radius focused spot itself. Additional simulations with different values of $k_{2}$ and $z$ intervals show a similar doughnut formation. Hence, the only necessary condition to achieve the desired spot is by melting a small region in which the extinction coefficient of the material is locally increased. Based on this fact, different materials that posses this feature can be potentially used as the active layer.

\section{Alternative Stack Layer Based on $\mathrm{AgO}_{x}$}

As an alternative for the $\mathrm{PC}$, a stack layer comprising a thin layer of $\mathrm{AgO}_{x}$ is numerically studied. The $\mathrm{AgO}_{x}$ is known to respond nonlinearly to a strongly focused spot above a certain power threshold [14]. In fact, the $\mathrm{AgO}_{x}$ thin film decomposes itself into a cluster rich in $\mathrm{Ag}$ after the oxygen is released due to the thermal energy. As a result, a dramatic increase in the extinction coefficient occurs. Specifically, the refractive index changes from $n=2.66+0.1 i$ in the $\mathrm{AgO}_{x}$ state to $n=0.7+3.65 i$ in the $\mathrm{Ag}$ state at $405 \mathrm{~nm}$ wavelength. Furthermore, this change occurs in a reversible manner, making the $\mathrm{AgO}_{x}$ a perfect candidate for the active layer. To evaluate this material, we have considered a stack layer consisting of a $20 \mathrm{~nm} \mathrm{AgO}_{x}$ layer, surrounded by two $\mathrm{ZnS}: \mathrm{SiO}_{2}$ dielectric layers, deposited on glass [14]. The field is computed using the model previously described. The advantage of using a thin layer of a nonlinear material with known thickness is that the height of the cylinder is no longer a variable but only its radius. In Fig. 8(a), the total electric field along the $x-z$ plane is shown for an $\mathrm{Ag}$ cluster scatterer of $120 \mathrm{~nm}$ radius. Similarly to the PC case, this stack layer also generates a welldefined dark region underneath the scatterer, indicating that the formation of the doughnut spot will also occur. Indeed, by plotting the focused spot computed along the dotted line in the plane $x-y$ [see Fig. 8(b)], the desired structured spot is found depending on the radius of the near-field scatterer, which in turn is related to the incident laser power. The particular spot computed with $120 \mathrm{~nm}$ scatterer, for instance, presents a diameter of $900 \mathrm{~nm}$ and a diameter of inner dark region of $400 \mathrm{~nm}$. The intensity ratio between the maximum intensity on the ring and the minimum intensity on the central dark region is $1: 0.1$. Considering the aforementioned properties of the $\mathrm{AgO}_{x}$ layer, we believe that this material is perfect to achieve the near-field self-induced doughnut spot.

\section{Conclusions}

In conclusion, we experimentally observed the doughnut spot generation in a PC/ZnS sample, after a high-power blue laser was focused onto the sample. The near-field measurements show that a stable hollow spot is formed at $3.5 \mathrm{~mW}$ laser power, as a result of the focused field interacting with an induced molten region. The doughnut spot formation mechanism was discussed based on the experimental evidences combined with a numerical model based on the FEM. Simulations show that indeed such a peculiar spot can be formed under specific circumstances. In fact, the only requirement necessary is a change in the refractive index in which the extinction coefficient becomes higher in the molten state. Therefore, other materials that posses this property could be used as the active media, as the simulations with $\mathrm{AgO}_{2}$ demonstrated. Hence, in this work we propose a new technique to obtain a doughnutlike focused spot without changing the laser beam polarization, establishing ideas that advanced microscopy, laser writing, and optical trapping could take advantage of.

We acknowledge support of this work by the FP7 European project IST-SURPASS, Project No. 224226. We thank CEA-LETI Minatec, Grenoble, France for preparing the samples.

\section{References}

1. S. W. Hell and J. Wichmann, "Breaking the diffraction resolution limit by stimulated emission: stimulated-emissiondepletion fluorescence microscopy," Opt. Lett. 19, 780-782 (1994).

2. J. Fischer and M. Wegener, "Three-dimensional direct laser writing inspired by stimulated-emission-depletion microscopy," Opt. Mater. Express 1, 614-624 (2011).

3. T. F. Scott, B. A. Kowalski, A. C. Sullivan, C. N. Bowman, and R. R. McLeod, "Two-color single-photon photoinitiation and photoinhibition for subdiffraction photolithography," Science 324, 913-917 (2009).

4. M. Dienerowitz, M. Mazilu, and K. Dholakia, "Optical manipulation of nanoparticles: a review," J. Nanophoton. 2, 021875 (2008).

5. D. Ganic, X. Gan, and M. Gu, "Optical trapping force with annular and doughnut laser beams based on vectorial diffraction," Opt. Express 13, 1260-1265 (2005).

6. Q. Zhan, "Cylindrical vector beams: from mathematical concepts to applications," Adv. Opt. Photon. 1, 1-57 (2009).

7. R. Dorn, S. Quabis, and G. Leuchs, "Sharper focus for a radially polarized light beam," Phys. Rev. Lett. 91, 233901 (2003).

8. D. Ganic, X. Gan, and M. Gu, "Focusing of doughnut laser beams by a high numerical-aperture objective in free space," Opt. Express 11, 2747-2752 (2003).

9. T. Hirayama, Y. Kozawa, T. Nakamura, and S. Sato, "Generation of a cylindrically symmetric, polarized laser beam with narrow linewidth and fine tunability," Opt. Express 14, 12839-12845 (2006).

10. A. J. Wachters and H. P. Urbach, Finite-element model for electromagnetic scattering problems, Tech. Note PR-TN 00042 (Phillips Research Europe, 2008).

11. X. Wei, A. J. Wachters, and H. P. Urbach, "Finite-element model for three-dimensional optical scattering problems," J. Opt. Soc. Am. A 24, 866-881 (2007).

12. A. van de Nes, L. Billy, S. Pereira, and J. Braat, "Calculation of the vectorial field distribution in a stratified focal region of a high numerical aperture imaging system," Opt. Express 12, 1281-1293 (2004).

13. A. C. Assafrao, A. J. H. Wachters, M. Verheijen, A. M. Nugrowati, S. F. Pereira, H. P. Urbach, M.-F. Armand, and S. Olivier, "Direct measurement of the near-field super resolved focused spot in InSb," Opt. Express 20, 10426-10437 (2012).

14. J. Tominaga and T. Nakano, Optical Near-Field Recording (Springer, 2004). 